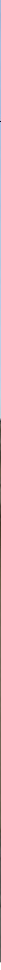

49. DDG-Tagung in Berlin

\title{
Jahreshighlight der Kongresssaison
}

Offensichtlich kam das Konzept 2015 gut an: Auch in diesem Jahr wurde bei der Tagung der Deutschen Dermalogischen Gesellschaft (DDG) vom 26.-29. April im Berliner City Cube in über 60 Kursen, Symposien und Plenarvorträgen versucht, die Neuerungen auf dem weiten Feld der Dermatologie abzudecken. Präsentiert und diskutiert wurden unter anderem kutane Nebenwirkungen der Immuntherapie, Biosimilars bei Psoriasis und die Zukunft molekularer Diagnostik von entzündlichen Dermatosen.

\section{Biosimilars: mehr Segen als Fluch}

Bi iosimilars bieten einen potenziell kostengünstigeren Zugang zur Versorgung mit biotechnologisch hergestellten Wirkstoffen. Doch Wirksamkeit und Sicherheit müssen aller Kostenkalkulation zum Trotz die entscheidenden Kriterien bleiben.

Biosimilars sind biotechnologisch hergestellte Arzneimittel, die dem Referenzbiologikum in der Struktur ähneln und daher für die gleiche Indikation zugelassen sind. Ein Austausch der Wirkstoffe ist nur dann möglich, wenn das Biosimilar seinem Referenzmedikament in puncto Wirksamkeit und Sicherheit gleicht. Wie Prof. Matthias Augustin, Hamburg, ausführte, stiegen die Ausgaben im Gesundheitswesen für Biologika in den letzten Jahren rasant an. Allein im Jahr 2013 beliefen sich die Kosten für Biologika in Deutschland auf 35 Milliarden Euro. Obwohl Biologika nur 2\% aller verschreibungspflichtigen Medikamente ausmachten, betrug ihr Anteil an den Gesamtkosten $21 \%$. Speziell für die Psoriasis ergab sich in den Jahren 2010 bis 2015 eine Zunahme des Arzneimittelumsatzes um $218 \%$, die monoklonalen Antikörper Adalimumab und Ustekinumab - und damit Biologika - waren im Jahr 2015 die umsatzstärksten Arzneimittel.

Die nun nach und nach auslaufenden Patente für die neuartigen Wirkstoffe bei der Therapie der Psoriasis erlauben die Vermarktung und den Einsatz von Biosimilars - die Preisdifferenz zum jeweiligen Referenzprodukt ist laut Au- gustin enorm, Einsparungen von bis zu $40 \%$ scheinen möglich. Bei einem flächendeckenden Einsatz von Biosimilars könnten schlussendlich deutlich mehr Patienten zu den gleichen Kosten behandelt werden. Bei den innerhalb der EU zugelassenen Biosimilars sei der Grad der „Similarität“ zum Referenzmedikament hoch.

Dennoch, so eine Forderung Augustins, müsse die Entscheidungshoheit beim verordnenden Dermatologen bleiben. So können gewichtige Gründe gegen den Wechsel eines Präparates sprechen. Der Nachweis von Bioäquivalenz, Qualität und Sicherheit des Biosimilars habe zudem stets Vorrang vor einem möglichen ökonomischen Nutzen.

Kathrin von Kieseritzky

S08/03: Biosimilars: Fluch oder Segen? 